\title{
Declarative Modeling for Machine Learning and Data Mining (Abstract)
}

\author{
Luc De Raedt
}

Department of Computer Science, Katholieke Universiteit Leuven, Belgium

\begin{abstract}
Despite the popularity of machine learning and data mining today, it remains challenging to develop applications and software that incorporates machine learning or data mining techniques. This is because machine learning and data mining have focussed on developing high-performance algorithms for solving particular tasks rather than on developing general principles and techniques.

I propose to alleviate these problems by applying the constraint programming methodology to machine learning and data mining and to specify machine learning and data mining problems as constraint satisfaction and optimization problems. What is essential is that the user be provided with a way to declaratively specify what the machine learning or data mining problem is rather than having to outline how that solution needs to be computed. This corresponds to a model + solverbased approach to machine learning and data mining, in which the user specifies the problem in a high level modeling language and the system automatically transforms such models into a format that can be used by a solver to efficiently generate a solution. This should be much easier for the user than having to implement or adapt an algorithm that computes a particular solution to a specific problem.

I shall illustrate this using our results on constraint programming for itemset mining [1] and probablistic programming. Some further ideas along these lines are contained in [2].
\end{abstract}

\section{References}

1. Guns, T., Nijssen, S., and De Raedt, L. (2011). Itemset mining: A constraint programming perspective. Artificial Intelligence, 175(12-13):1951 - 1983.

2. De Raedt, L., and Nijssen, S. (2011). Towards Programming Languages for Machine Learning and Data Mining (Extended Abstract). Proceedings of the 19th International Symposium on Foundations of Intelligent Systems, Lectures Notes in Computer Science, Vol. 6804, pp. 25-32. 